\title{
Collaborative research exploring mental health service user perspectives on acute inpatient occupational therapy.
}

\begin{tabular}{|c|c|}
\hline Journal: & British Journal of Occupational Therapy \\
\hline Manuscript ID & 079-Apr-2015-RP.R2 \\
\hline Manuscript Type: & Research Paper \\
\hline Key Areas: & $\begin{array}{l}\text { Adult Psychosocial < Clinical, Research Methods and Methodology, Theory } \\
\text { and Philosophy }\end{array}$ \\
\hline Keywords: & photo-elicitation, user involvement, occupational engagement \\
\hline Abstract: & $\begin{array}{l}\text { Introduction: User perspectives are important for understanding why } \\
\text { people engage with occupational therapy during an admission for acute } \\
\text { mental health issues, to inform service provision and development. } \\
\text { Method: } 22 \text { recent and current inpatients participated in } 6 \text { semi-structured } \\
\text { individual interviews and } 3 \text { focus groups. Data from the two methods were } \\
\text { initially subject to separate thematic analysis. Then a further stage of } \\
\text { constant comparative analysis, of both data sets, generated the findings } \\
\text { presented here. } \\
\text { Findings: Three themes were identified: (1) "A tiny sort of world" } \\
\text { expressed experiences of being restricted; (2) "Relief" indicated how } \\
\text { occupational therapy offered relief from the ward and experiences of } \\
\text { mental ill-health; and ( } 3 \text { ) "Something to do" suggested specific purposes } \\
\text { for engaging in occupation. These themes indicate how service users } \\
\text { experience and value occupational therapy for different reasons at different } \\
\text { times. The approach of occupational therapists to service users, valuing } \\
\text { them as occupational beings, is a key aspect of their experience. } \\
\text { Conclusion: The profession is challenged to design flexible opportunities for } \\
\text { occupational engagement, which simultaneously provide relief and } \\
\text { distraction, address diverse occupational needs, and are feasible within the } \\
\text { resource restrictions of acute mental health services. }\end{array}$ \\
\hline
\end{tabular}


Perspectives on acute inpatient mental health occupational therapy

Collaborative research exploring mental health service user perspectives on acute inpatient occupational therapy.

Key words: photo-elicitation, user involvement, occupational engagement, recovery

\begin{abstract}
Introduction: User perspectives are important for understanding why people engage with occupational therapy during an admission for acute mental health issues, to inform service provision and development.
\end{abstract}

Method: 22 recent and current inpatients participated in 6 semi-structured individual interviews and 3 focus groups. Data from the two methods were initially subject to separate thematic analysis. Then a further stage of constant comparative analysis, of both data sets, generated the findings presented here.

Findings: Three themes were identified: (1) "A tiny sort of world" expressed experiences of being restricted; (2) "Relief" indicated how occupational therapy offered relief from the ward and experiences of mental ill-health; and (3) "Something to do" suggested specific purposes for engaging in occupation. These themes indicate how service users experience and value occupational therapy for different reasons at different times. The approach of occupational therapists to service users, valuing them as occupational beings, is a key aspect of their experience.

Conclusion: The profession is challenged to design flexible opportunities for occupational engagement, which simultaneously provide relief and distraction, address diverse occupational needs, and are feasible within the resource restrictions of acute mental health services. 
Perspectives on acute inpatient mental health occupational therapy

\section{Introduction}

The perspectives of people with acute mental health problems on their experience of occupational therapy as inpatients are not strongly evident in the literature. In the UK, community mental health services have expanded in recent years to address acute problems, with a parallel reduction in inpatient beds (Glover et al 2006). As a core mental health profession, occupational therapy has evolved in response, aiming to maintain safety while also promoting the recovery approach (National Institute for Mental Health in England (2007). The impact of these changes could be understood by engaging with service user perspectives (Nolan et al 2011). This research was initiated by occupational therapists inspired by previous local studies involving service users (refs removed for anonymity: 1,2). The occupational therapists were based in an occupational therapy department in an acute unit on a large general hospital site in London, UK, which had three wards including an intensive care unit. They formed a steering group, which over five years involved service user researchers, practitioners, academics and MSc students. They started working collaboratively by identifying their shared values, which shaped review of relevant literature as the research progressed.

\section{The challenges of the inpatient setting}

Intervention studies, such as Killaspy et al (2014), have evaluated the effectiveness of occupational therapy in inpatient rehabilitation services, but with inconclusive findings. Generic manualised interventions have also been developed and evaluated collaboratively, with varied success (Chen et al 2013). The acute inpatient setting presents particular challenges for manualised interventions and associated studies, because of the need to simultaneously keep people safe, assess needs, meet basic care needs and provide physical healthcare and activities (Bowers et al 2009). Occupational therapists have to engage with rapidly fluctuating needs by working with individuals and creating group-based opportunities (Lloyd and Williams 2010, ref removed for anonymity 3). The recovery approach has also been influential and to some extent is aligned with a social perspective on mental health, emphasising external factors which influence recovery, particularly attitudinal barriers from staff and the wider community (Chen et al 2013). However, specific challenges arise within acute units from the fast-changing interactions between these external factors and the internal world of people in an acute phase of mental ill health (Creek 2010). When this research was initiated, the most relevant published studies did not adequately reflect current acute service provision (College of Occupational Therapists 2006). Only Lim et al (2007) had collaborated with service user researchers, but qualitative data was limited.

\section{The service user voice}

Studies by occupational therapists have indicated that service users have valued many aspects: occupational therapists' approaches (College of Occupational Therapists 2006); the social and physical environment for occupational therapy (Haley and McKay, 2004); and the interventions used (Lim et al 2007). Occupational therapy has been successful in motivating people by engaging them in an environment which offered structure, respect, support, a change from the ward and occupations which were not otherwise accessible (Haley and McKay 2004). However, the lack of recent research meant it was not possible to know whether service user perspectives have changed as services have 
Perspectives on acute inpatient mental health occupational therapy

evolved, especially over recent years when there have been more compulsory admissions (Health and Social Care Information Centre 2014).

\section{Engaging with users through collaborative research}

A range of research methods have been used including self-report questionnaires (Lim et al 2007) and semi-structured interviews (Haley and McKay 2004). However, these studies involved service users primarily as participants rather than co-researchers. Collaborative research, undertaken by partnerships between service users, practitioners and academic researchers, can enable research ideas to emerge and develop from experiences of both using and delivering services (Beebe et al 2012). This research aimed to explore how acute inpatient mental health occupational therapy services were perceived by service users. Analysis of these perspectives could then inform local service development and wider understandings of occupational therapy in this setting.

\section{Method}

A qualitative approach was used, informed by an ethnographic focus which was concerned with experiences of a particular place or culture, and an emancipatory purpose, to give voice to marginalised experiences and create knowledge which could be used to inform service improvements (Papadimitriou et al 2012). The research was undertaken collaboratively by a team with changing membership over the five years duration. Drawing on previous audits, the literature and other sources of practice knowledge, a topic guide was agreed (Reagon et al 2010). The topic guide covered length of stay, sessions attended, reasons for not attending, helpful groups, suggestions for groups, individual occupational therapy, outreach work, and finally suggestions for improvement. Focus groups and semi structured interviews were used for data gathering. These methods were accessible and practical, giving participants a realistic choice about how to participate. Two service user researchers were involved in participant recruitment and facilitated the focus groups with an MSc occupational therapy student. Another student conducted the interviews. It was believed that participants could express themselves more freely to independent service user and student researchers, enabling a deeper understanding to be gained.

Inclusion criteria were as follows: all current in-patients and people discharged within the last 6 months preceding recruitment; people of any ethnic origin between the ages of 18 and 70; formal (those on sections of the Mental Health Act 2007) and informal status; and able to communicate in English. Exclusion criteria were: those on the detoxification programme or in the psychiatric intensive care unit; those on close observations at the time of data gathering; and those who lived outside the local area.

Posters and leaflets were produced, displayed on the unit and distributed in ward community meetings. The hospital database was used to identify previous service users, who were sent information about the research and then followed up. Everyone who expressed an interest and met the inclusion criteria was included. The research was explained to participants on the day of data gathering to inform the consent process. All those who consented to participate were given $f 18$, Version 260415 
meeting the local policy for service user involvement payments. Interview and focus group questions were initially developed by the students, using the topic guide. They piloted these questions with the service user researchers. Some questions were modified or substituted. This process gave the students and service users gained-a valuable collaborative opportunity to experience, reflect and prepare for data gathering. To elicit richer responses, the photo elicitation method was used (Harper 2002). Photographs of rooms in the occupational therapy department were taken in advance by the service user researchers, including the kitchen, gym, art room and internet café. A list of the usual group sessions was also available as a prompt. All the interviews took place in group meeting rooms in the occupational therapy department. The focus groups took place in a separate meeting room and refreshments were provided during the groups, which lasted up to 90 minutes.

\section{Data analysis}

The focus groups and interviews were digitally recorded and transcribed verbatim by the students. They, who initially and_separately_conducted thematic analyses of the data they had collected, and their field notes. They presented their findings to the steering group, who observed that a further stage of analysis of both sets of data together might generate deeper insights, using comprehensive constant comparative analysis. This was done by the chief investigator and two new occupational therapists in the team, who had been appointed after the data had been gathered. It was a highly reflexive process, requiring the researchers to critically scrutinise the data and their own experience of working in similar settings (Silverman 2005). With regular discussion in the regular steering group meetings, this stage of analysis enabled further consideration of different perspectives on the participants' experiences and theorising about the findings.

\section{Ethics}

Approval was obtained from the university, the local NHS Ethics committee and the local Research \& Development Board. Data were stored securely at the university to ensure confidentiality. The engoing close collaboration ensured that the research was timedData collection was scheduled to workffit with the unit's routines and priorities, to avoid disrupting occupational therapy service provision. It was supported with back up from the staff as required.

\section{Trustworthiness}

This research involved different people at each stage, which could have undermined the overall coherence if roles had not been clearly agreed at each stage by the service manager and chief investigator, who were consistently involved throughout. This inclusive approach generated opportunities to make choices about involvement in specific research tasks and activities, reflecting an occupational justice approach (Stadnyk et al 2011, removed for anonymity: 2). Although the service user researchers were not involved before ethical approval was obtained, recruitment and data gathering via the focus groups were enhanced by their involvement, with a more rigorous and accessible approach to service user participants. The steering group had a range and depth of experience, enabling peer review and adding credibility to the data gathering and analysis. Having students involved generated another perspective, as they were not familiar with the service. Photoelicitation techniques successfully prompted memories, especially for those participants who had been recently discharged, adding to the richness of the data. 
Perspectives on acute inpatient mental health occupational therapy

\section{Findings}

There were 22 participants in six semi structured interviews (three men and three women) and three focus groups (12 men and four women). To simplify the experience of participating in the research, given their acute mental health issues, they were not required to fill out demographic information forms. Researcher observations indicated that participants' ages ranged from 18 to 64 and they were from a range of ethnic backgrounds, reflecting the demographic profile of the local area.there were participants from Black and South Asian minority ethnic groups and white British populations. Specific information about diagnoses was not available as this is often still uncertain in acute settings. There was a mix of current and former service users, with all experiencing admission in the past six months and therefore recent experience of the unit.

There were three themes emerging from the second stage of data analysis, tracking user journeys through the occupational therapy service. The first theme, "A tiny sort of world," expressed experiences of being restricted on the ward and by internal experiences of mental health problems. "Relief", the second theme, indicated how occupational therapy offered an opportunity to get away from the ward. "Something to do" suggested the individual purposes for engaging in occupation. These three themes also included criticisms of acute services. Extracts from the data have been used to support the themes, identified by focus group (FG) or interview (INT), and pseudonyms.

\section{"A tiny sort of world": being on the ward}

From the service user perspective, being on the ward meant being restricted in many ways, not having much to do and being monitored. Participants were aware of this focus for staff:

"I'm trying to sleep and he has to bash in and wake you up. I looked at him and I said what are you doing? and he said I'm checking whether you are asleep."(Mark, FG1)

"You told them at the office, look, I'm just leaving can you open the door? They still take a good five, ten minutes to open the door, so as soon as that door's open, ... "whew"' (Abhu, FG1)

There was a parallel between this restricted environment and the internal experience of mental health problems:

"When you're ill, you're sort of in a tiny sort of world." (Mel, FG3).

"When I was in my room there was nothing to do ... I was just sitting there looking at the four walls for ages." (Nicole, INT4).

Participants knew there were implications of these restrictions for their health:

"You can't expect to be locked up 24 hours on the ward and expect to be healthy." (Neil, INT1)

"If you're stuck in one place then you get depressed." (Aalok, FG3)

Although the negative aspects of the ward environment were mentioned by most participants, others recognised that they needed time to overcome their own mental health problems: 


\title{
British Journal of Occupational Therapy
}

Perspectives on acute inpatient mental health occupational therapy

\begin{abstract}
"It takes time for people, [for] their medication to actually work and to start thinking about going to these groups" (David, FG2)

\section{"Relief": being away from the ward}

Leaving the ward environment was seen as a primary benefit of going to the occupational therapy department:

"Colin: For me it's mainly to escape from the ward

Mel: Yea and me

Freya: Yea and me." (FG3)

For some the sense of relief was from tension and a focus on problems, especially in the gym where people could "let off some steam" (Becky and Abhu FG1):

"Definitely getting out and about is a great relief isn't it?" (David, FG2)

Some of the relief which participants experienced could be viewed as transient:

"I had a [hand] massage because I felt too distressed that day and the OT made sure I was relaxed." (Catherine, INT3)

This sense of relief is understandable: from the service user perspective, distraction and diversion have a serious purpose:

"Get off the ward to be occupied and think about other things apart from illness." (Catherine, INT3)

These experiences were dependent on the therapists' approach and priorities. For Becky, the opportunity to cook was important and she was frustrated by not having this option:

"they said it was for people who need to learn and cause they said I was well I didn't need to be here doing it, which I thought was a bit unfair" (Becky, FG1)

Access to and information about occupational therapy was sometimes a problem, with some participants not being able to access it at all, and another having to wait to be taken to occupational therapy, which he found humiliating, making him "feel like a lemon" (Alan, FG2).

Participants suggested that occupational therapy should be more accessible:

"It should be open ... more available really" (Becky, FG1)

One participant did not manage to access occupational therapy during one very short admission and commented that "... doing any of them [therapy sessions] would have helped..." (Nicole, INT4).

\section{"Something to do": expressing occupational needs}

This fundamental sense of the importance of doing something was shared and discussed by participants, recognising the benefits of occupation: 
Perspectives on acute inpatient mental health occupational therapy

"I just have [to have] something to do ... something different." (Neil, INT1)

"they said here is a smoke break or fresh air breaks, but you want more than that sometimes."(Sid, FG2)

All participants were encouraged to reflect on specific groups, prompted by the photographs and list provided:

"I couldn't put it into context why I found them beneficial but they all got different bits which have helped me quite a bit. I still feel low, I still feel depressed, but I would have felt worse" (Alan, FG2)

"Facilitator: OK, so which ones [sessions] do people find most helpful?

Scott: Football

Facilitator: Why, why's that helpful?

Scott: Stress relief

Abhu: Yea" (FG1)

"...yoga was good because it got you moving your body..." (Catherine, INT3)

"I like creative writing, gives you a chance to express yourself."(Anna, FG1)

Not everyone found sessions helpful, particularly if the occupation did not appeal to them.

"I don't see the point of it you know. What are you going to get out of drawing a picture?" (Anna, FG1)

The group sessions offered opportunities for social interaction, particularly between genders as they came from separate wards:

"I think the room is a little bit squashy, you know like 7 computers and when it is full [we are] bumping elbows ... I like the computer [session], part of it catching up on your email obviously and that it was mixed male and female" (Helen, INT2)

Being offered a choice about whether to join a session was important.

"The OT came down ... half a week after I was admitted and sat with me and went through the activities on the sheet and we circled which ones I was interested in doing" (Helen, INT2)

"you get to do something which you like, you get to do it yourself" (Abhu, FG1)

Many participants observed that the approach of occupational therapy staff was most important and participants in the first focus group spent some time discussing this:

"they're more polite and they make you feel welcome, the majority of them."(Becky, FG1)

"they try to encourage you" (Anna, FG1) 
Perspectives on acute inpatient mental health occupational therapy

Participants valued the occupational therapists' approach in contrast to how they were treated elsewhere. Abhu observed that the ward atmosphere changed when occupational therapy staff were present:

"cause OT lot, they do come in and as soon as OT lot come in you just see everyone's behaviour's changed."(Abhu, FG1)

"some staff look down on you but people downstairs [in the occupational therapy department] don't, they treat you as an equal." (Scott, FG1)

"they talk to you like a normal person and so your attitude changes so much quicker."(Abhu, FG1)

It is difficult to appreciate what is important to a person without exploring their occupational needs, illustrated by one participant who was frustrated with a doctor's emphasis on the benefits of occupational therapy in a very general sense.

"the doctor bangs on about OT and how good it is for you, but if you don't enjoy doing things you're not going to go" (Anna, FG1)

In contrast, Neil's perspective was focused on the environment for occupational therapy, in the garden:

"Getting fresh air, like real fresh air" (Neil, INT1)

This contrast between occupational therapy and the ward environment can be discussed in a number of ways.

\section{Discussion}

The participants shared their experiences of the ward environment and occupational therapy, suggesting that their journeys through the service mirrored internal shifts in their mental health. As they recovered, they were able to move from a restricted environment with relief, and engage with occupation as a therapeutic medium in the separate occupational therapy department, which contrasted with other local units where occupational therapy resources were much more restricted. Lloyd and Williams (2010) suggest in their critical review that research has not given sufficient focus to the whole experience of occupational therapy, proposing four core elements of practice from assessment to discharge planning, much of which occurs beyond the specific sessions often identified as occupational therapy. While these elements are not new, they articulate the full potential of occupational therapy, reflecting diverse experiences of occupational therapy. The findings of this research offer further insight into these elements, from service user perspectives.

Initial analysis of the data informed immediate changes to the occupational therapy service. Urgent attention was given to how information could be shared more effectively so that service users were not left "feeling like a lemon" if sessions were cancelled at short notice. The findings indicated how occupational therapy could be part of a person's progress through the service, valuing early contact, offering relief from the ward and echoing the idea of a recovery journey. However, perceiving recovery as a journey can have also limitations, especially within a short admission, suggesting a smooth progress to a destination which in reality has twists and turns and setbacks (Kelly et al 2010). 
Further analysis indicated that for some participants, occupational therapy was a dynamic part of the interface between the external world of the unit and their internal world, disrupted by experiences of acute mental health problems (Creek 2010). The impact of occupational engagement on the internal experience of acute mental health problems was indicated by participants, who recognized how being distracted from distressing thoughts and sights enabled them to feel "a better person". To some extent this challenges a longstanding concern of occupational therapists that activities which emphasise distraction, diversion or entertainment undermine the serious purpose of occupational therapy (Dewis and Harrison 2008). The participants indicated that it was important to be distracted or diverted from their internal worlds at the beginning of their journey. This suggests the importance of a graded process, where progress might be evident with people making an active choice about their occupational needs, encouraged by occupational therapists and other staff. It may also be important to accept that many people will see the primary benefit of occupational therapy as being a distraction, because of the short duration of admissions. Cohesive links between inpatient and community occupational therapy should ensure occupational needs are addressed in a sustained approach.

The priority given to safety and monitoring in acute inpatient units is difficult to question, yet it is a concern that some practices may overshadow service user needs (Wood \& Pistrang 2004). The ward setting impacts on everyone there, including ward-based staff, especially if it is locked (Ashmore 2008). This was indicated by Abhu's observation that the whole atmosphere seemed to change when occupational therapists were present. Staff who do not have a positive view of the future for service users can undermine the recovery process (Wood \& Pistrang 2004). This would seem more likely in an environment which is highly restrictive and therefore gives less opportunity for people to demonstrate positive capacities, reflecting service priorities for safety and monitoring but not acknowledging what might actively promote mental wellbeing (Shepherd et al 2008). It could also be argued that an occupational perspective directly challenges the emphasis on safety and monitoring. Participants shared how it was important to have experiences beyond the ward and beyond themselves, echoing ongoing concerns about the absence of things to do (Department of Health 2002). However, being bored can signify much more than a lack of things to do, as the struggle to engage with occupations is most likely due to a combination of acute mental health problems and the ward environment (Bowers et al 2009). If the ward environment is associated with occupational deprivation, which would echo findings in similar settings such as prisons (Stadnyk et al 2011), then it would appear to make sense to provide more opportunities for occupational engagement within the ward itself.

However, occupational engagement is 'a sense of involvement, choice, positive meaning and commitment while performing an occupation or activity' (Creek 2010 p166), enabling people to actively influence their health and well-being (Doble and Caron Santha 2008). Diverse opportunities are critically important. For example Neil dismissed the "fresh air breaks" as not being enough and highlighted the importance of "real" fresh air for him, suggesting that getting fresh air had occupational and social significance over and above the physical act of breathing outside. Addressing diversity is about understanding these subtle differences in perspective. Thus it was the focus and approach of occupational therapists that made a difference, not just the provision of activities. This suggests a limit to the usefulness of solely providing activities on the ward... 
Perspectives on acute inpatient mental health occupational therapy

Doble and Caron Santha (2008) argue that to promote wellbeing, occupational therapists need to shift their focus from occupational performance to how individuals experience and "orchestrate" their occupational lives. Specifically relevant to this research is the idea of renewal as a route to wellbeing, where people are refreshed from engaging in one occupation to the extent they can face engaging in another. Alan (FG2) indicated this with his perception of the combined effects of occupational therapy sessions, not being able to isolate specific examples but knowing the overall experience had been beneficial. Thus well-being is not just derived from outcomes of sessions but is also part of the process of engagement (Wilcock 2006). This suggests an area for further inquiry into the impact of both transient and sustained experiences of occupational engagement and their relationship to mental health and wellbeing, particularly in relation to the idea of renewal and relief (Doble and Santha 2008).

The collaboration which enabled this research to take place acknowledged diverse occupational needs of the staff: waiting for a stable team and academic availability would have prevented it from occurring at all. The flexible approach was based on a pragmatic approach to research, recognizing that knowledge and understanding can be developed over a prolonged period. The experience of the research as an occupation enhanced both the practice and the academic environments, as a resource for learning and building collaborative relationships. This approach reflects understandings of adult learning and the scholarship of practice (Forsyth et al 2005).

\section{Limitations}

The two methods of data gathering addressed some limitations of earlier research in this area, but were dependent on participants being able to share their thoughts and opinions verbally and coherently. Some findings could be used immediately to improve services, but initial data analysis suggested that participants had primarily focused on group work, causing steering group members to question whether other aspects of occupational therapy had been discussed. Deeper analysis of both data sets suggested a broader focus. Further research involving participants over several weeks, rather than a one-off session, could strengthen the findings here.

\section{Conclusion}

This research has offered insights into service user perspectives of occupational therapy in an acute mental health unit. Occupation provides important relief from the ward environment and from service users' internal worlds, in an acute episode. This experience of relief was gained by leaving the ward, an experience which may be restricted in units where occupational therapists do not have separate facilities. Participants recognised the importance of having choices about what to do for their own recovery, and valued the approach of occupational therapists in providing this. There is a continuing challenge for the profession to design flexible opportunities for occupational engagement which provide relief and distraction, address diverse occupational needs, and are feasible within the resource restrictions of acute mental health services. Further investigation, within collaborative research partnerships, could offer more detailed understandings from theory and practice.

4176 words

Version 260415 
Perspectives on acute inpatient mental health occupational therapy

2

3

4

5

6

7

8

9

10

11

12

13

14

15

16

17

18

19

20

21

22

23

24

25

26

27

28

29

30

31

32

33

34

35

36

37

38

39

40

41

42

43

44

45

46

47

48

49

50

51

52

53

54

55

56

57

58

59

60

\section{Key findings:}

1. Acute mental health ward environments restrict occupational engagement, with implications for health and well-being.

2. An occupational therapy department provides relief and opportunities for recovery.

3. The approach of occupational therapists is important for occupational engagement.

What the study has added: 30 words

Opportunities to engage in occupational therapy are valued by acute mental health service users for diverse reasons, indicating the importance of enabling occupation to promote health and wellbeing. 


\section{British Journal of Occupational Therapy}

Perspectives on acute inpatient mental health occupational therapy

References (numbered references removed for anonymity)

Ashmore R (2008) Nurses' accounts of locked ward doors: ghosts of the asylum or acute care in the $21^{\text {st }}$ century? Journal of Psychiatric and Mental Health Nursing 15, 175-185

Beebe L, Smith K, Davis J, Roman M, Burke R (2012) Meet me at the crossroads: clinical research engages practitioners, educators, students and patients. Perspectives in Psychiatric Care 48: 76-82

Bowers L, Chaplin R, Quirk A, Lelliott P (2009) A conceptual model of the aims and functions of acute inpatient psychiatry. Journal of Mental Health, 18 (4): 316-325.

Chen S, Krupa T, Lysaght R, McCay E, Piat M (2013) The development of recovery competencies for in-patient mental health providers working with people with serious mental illness. Administration and Policy in Mental Health and Mental Health Services Research 40, (2): pp 96-116

College of Occupational Therapists (2006) Recovering Ordinary Lives: The Strategy for Occupational Therapy in Mental health Services 2007-2017, Results from Service User and Carer Focus Groups (Core). London: College of Occupational Therapists

Creek J (2010) The Core Concepts of Occupational Therapy. London: Jessica Kingsley Publishers.

Department of Health (2002) The Mental Health Policy Implementation Guide: Adult Acute Inpatient Care Provision. London: Department of Health.

Dewis S, Harrison M (2008) Engaging the disengaged. Practising in acute settings. In McKay EA, Craik C, Lim KH, Richards G (eds) Advancing Occupational Therapy in Mental Health Practice. Oxford, Blackwell Publishing.

Doble SE, Caron Santha J (2008) Occupational well-being: rethinking occupational therapy outcomes. Canadian Journal of Occupational Therapy 75(3), 184-190.

Forsyth K, Summerfield Mann L, Kielhofner G (2005) Scholarship of practice: making occupationfocused, theory-driven, evidence-based practice a reality. British Journal of Occupational Therapy, $68,6,260-8$

Glover G, Arts G, Babu KS (2006) Crisis resolution/home treatment teams and psychiatric admission rates in England. British Journal of Psychiatry, 189 (5): 441-445

Haley L, McKay EA (2004) Baking gives you confidence: users' views of engaging in the occupation of baking. British Journal of Occupational Therapy, 67(3), 125-128.

Harper D (2002) Talking about pictures: A case for photo elicitation. Visual Studies. 17(1), 13-24.

Health and Social Care Information Centre (2014) Inpatients formally detained in hospitals under the Mental health Act 1983, and patients subject to supervised community treatment. www.hscic.gov.uk accessed 12/04/2015

Kelly M, Lamont S, Brunero S (2010) An occupational perspective of the recovery journey in mental health. British Journal of Occupational Therapy, 73(3), 129-135. 
Perspectives on acute inpatient mental health occupational therapy

Killaspy H, Marston L, Green N, Harrison I, Lean M, Cook S, Mundy T, Craig T, Holloway F, Leavey G, Koeser L, McCrone P, Arbuthnott M, Omar R, King M (2014) Clinical effectiveness of a staff training intervention in mental health inpatient rehabilitation units designed to increase patients' engagement in activities (the Rehabilitation Effectiveness for Activities for Life (REAL) study): singleblind, cluster-randomised controlled trial. The Lancet, published online December 16, 2014.

Lim KH, Morris J, Craik C (2007) Inpatients perspectives of occupational therapy in acute mental health. Australian Occupational Therapy Journal, 54(1), 22-32.

Lloyd C, Williams PL (2010) Occupational therapy in the modern adult acute mental health setting: a review of current practice. International Journal of Therapy and Rehabilitation, 17(9), 436-442.

National Institute for Health and Clinical Excellence (2011) Service user experience in adult mental health: improving the experiences of care for people using adult NHS mental health services. Manchester: National Institute for Health and Clinical Excellence

National Institute for Mental Health in England (NIHME) (2007) New ways of working for everyone. London: NIHME Workforce Development Programme.

Nolan P, Bradley E, Brimblecombe N (2011) Disengaging from acute inpatient psychiatric care: a description of service users' experiences and views. Journal of Psychiatric and Mental Health Nursing $18,359-367$

Papadimitriou C, Magasi S, Frank G (2012) Current thinking in qualitative research: evidence-based practice, moral philosophies, and political struggle. OTJR: Occupation, Participation and Health 32 (1) S2-5

Reagon C, Bellin W, Boniface G (2010) Challenging the dominant voice: the multiple evidence sources of occupational therapy. British Journal of Occupational Therapy, 73, (6), 284-286(3)

Shepherd G, Boardman J, Slade M (2008) Making Recovery a Reality. United Kingdom: Sainsbury Centre for Mental Health.

Silverman D (2005) Doing Qualitative Research. London: Sage.

Stadnyk RL, Townsend EA, Wilcock AA (2011) Occupational Justice. In Christiansen CH, Townsend EA, eds. Introduction to Occupation: The Art and Science of Living. $2^{\text {nd }}$ ed. New Jersey: Pearson Education Inc.

Wilcock AA (2006) An Occupational Perspective of Health. 2nd ed. Thorofare, NJ: Slack.

Wood D, Pistrang N (2004) A safe place? Service users' experiences of an acute mental health ward. Journal of Community \& Applied Social Psychology 14: 16-28 